FERMILAB-CONF-20-008-ND

PREPARED FOR SUBMISSION TO JINST

LIDINE 2019

AUG 28TH TO 30TH 2019

UNIVERSITY OF MANCHESTER

\title{
First calorimetric energy reconstruction of beam events with ARAPUCA light detector in protoDUNE-SP
}

\author{
D.Totani, ${ }^{a, b}$ F. Cavanna, ${ }^{b}$ on behalf of DUNE collaboration. \\ ${ }^{a}$ University of L'Aquila, L'Aquila, 67100, Italy \\ ${ }^{b}$ Fermi National Accelerator Laboratory, Batavia, Illinois 60510, USA \\ E-mail: dante.totani@aquila.infn.it
}

\begin{abstract}
ProtoDUNE Single Phase at CERN is the large scale prototype for the far detector of the future DUNE experiment. ProtoDUNE is in stable operation since Oct. 2018 at the CERN Neutrino Platform. Test beam data in the energy range of sub-GeV to a few $\mathrm{GeV}$ were collected in fall 2018 providing a set of key measurements. Particles (electrons, protons, pions, muons and kaons) are identified combining information from a set of beam-line detectors (Time of Flight, Cherenkov) and TPC reconstruction. Three different technologies are implemented in the protoDUNE photon detector system (PDS). Results from the ARAPUCA response, one of the PDS components, are presented providing first calorimetric energy measurements and particle ID of beam events from LAr scintillation light signals.
\end{abstract}

KEYwords: Neutrino detectors, Large detector systems for particle and astroparticle physics, Noble liquid detectors,

This document was prepared by [DUNE Collaboration] using the resources of the Fermi National Accelerator Laboratory (Fermilab), a U.S. Department of Energy, Office of Science, HEP User Facility. Fermilab is managed by Fermi Research Alliance, LLC (FRA), acting under Contract No. DE-AC02-07CH11359. 


\section{Contents}

1 The ProtoDUNE-Single Phase detector 1

1.1 The ARAPUCA PD modules 2

2 H4-VLE Beam Line Instrumentation 2

2.1 Particle Identification 3

3 Photon Detector Performance $\quad 4$

4 ARAPUCA Photon Detector Response to Beam Electrons 4

4.1 Linearity 5

4.1.1 Geometrical Corrections 6

4.2 Resolution 8

5 Conclusion 10

\section{The ProtoDUNE-Single Phase detector}

ProtoDUNE-SP is the single-phase DUNE Far Detector [1] prototype that is located at the CERN Neutrino Platform (NP) and it is in stable operation since Oct. 2018. ProtoDUNE-SP prototypes the designs of most of the single-phase DUNE far detector module (DUNE-SP) components at a 1:1 scale, with an extrapolation of about 1:20 in total LAr mass. The detector elements, consisting of the time projection chamber (TPC), the cold electronics (CE), and the photon detection system (PDS), are housed in a cryostat that contains the LAr target material. The cryostat, a free-standing steel-framed vessel with an insulated double membrane, is based on the technology used for liquefied natural gas (LNG) storage and transport. A cryogenics system maintains the LAr at a stable temperature of about $89 \mathrm{~K}$ and at the required purity level through a closed-loop process that recovers the evaporated argon, recondenses and filters it, and returns it to the cryostat. The ProtoDUNE-SP TPC, comprises two drift volumes, defined by a central cathode plane that is flanked by two anode planes, each at a distance of $3.6 \mathrm{~m}$, and a field cage (FC) that surrounds the entire active volume. The active volume is $6 \mathrm{~m}$ high, $7 \mathrm{~m}$ wide and $7.2 \mathrm{~m}$ deep (along the drift direction). Each anode plane is constructed of three adjacent Anode Plane Assemblies (APAs) that are each $6 \mathrm{~m}$ high by $2.3 \mathrm{~m}$ wide in the installed position. Each APA consists of a frame that holds three parallel planes of sense and shielding wires; the wires of each plane are oriented at different angles with respect to those on the other planes to enable 3D reconstruction. The wire pitch for all wire planes is $4.5 \mathrm{~mm}$, and each APA holds a total of 2,560 wires. The protoDUNE-SP photon detector system (PDS) is made of 60 optical modules embedded within the 6 APA frames of the TPC and thus viewing at the LAr volume from each anode side opposite to the central cathode. Three different photon collection technologies are utilized for the PDS modules. Each is 
designed to convert incident LAr scintillation photons (around $128 \mathrm{~nm}$, in the Vacuum-UVrange) into longer wavelength photons using photofluorescent compounds as wavelength shifters(WLS). Visible light is trapped within the module, a portion of which is eventually incident on an array of silicon photomultiplier photo-sensors.

\subsection{The ARAPUCA PD modules}

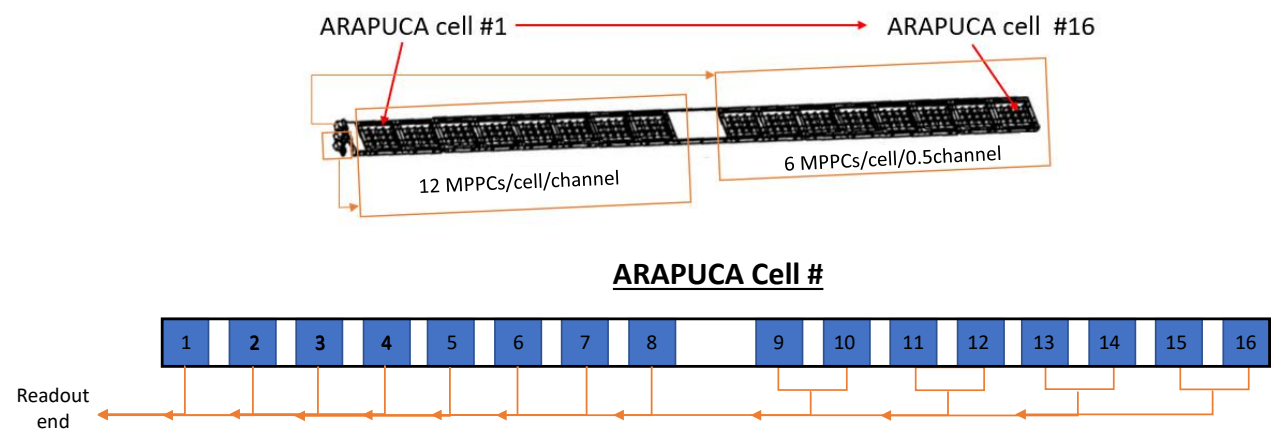

Figure 1. ARAPUCA PD moudule. The detector is organized in two groups of cells. The first 8 cells host 12 MPPC devices and each cell is read by a single channel. The second group of 8 sub-cells host 6 MPPC devices each one and a single channel reads two sub-cells. As result we have 12 channels, each one read 12 MPPC, arranged in 8 cells of $9.8 \times 7.9 \mathrm{~cm}^{2}$ and 4 cells of $2 \times 9.8 \times 7.9 \mathrm{~cm}^{2}$ sensitive area.

Two of the 60 PD modules are ARAPUCA modules [2]. One is located in the top half of the upstream APA in the beam side drift volume. A second is located in the middle of the APA in the center of the opposite drift volume. The ARAPUCA modules are segmented longitudinally in 12 cells with independent read-out, 8 with $9.8 \times 7.9 \mathrm{~cm}^{2}$ optical area and 4 with double area $\left(19.6 \times 7.9 \mathrm{~cm}^{2}\right)$. The ARAPUCA cells convert incident VUV photons into the visible range using pTP (p-terphenile, $\sim 350 \mathrm{~nm}$ ) as first WLS, then a dichroic filter window (400 $\mathrm{nm}$ cutoff) reflects photons from a second WLS (TPB, tetra-phenilbutadiene, $\sim 430 \mathrm{~nm}$ ) inside the cell underneath the window and prevent them from exiting until detected by the photo-sensors distributed inside the cell. Each cell of the ARAPUCA modules has $12\left(6 \times 6 \mathrm{~mm}^{2}\right)$ Silicon photo-sensors of Hamamatsu MPPCS13360-6050 (50 $\mu \mathrm{m}$ pixel size) - the CQ-type (Quartz windowed for Cryogenic application), distributed in the plane opposite to the cell optical window, for a total of 144 photo-sensors in the ARAPUCA module (12 cells). Each cell of the ARAPUCA modules is read-out by one channel (12 MPPC per channel). The readout system transmits unamplified signals from the photo-sensors in LAr to outside the cryostat on cables, and performs processing and waveform digitization. Based on the photon detector system requirements, a dedicated custom module was built for receiving silicon photo-sensor signals, and performing signal processing in the front-end as preprocessing for trigger and DAQ. The module is called the SiPM Signal Processor (SSP) [3].

\section{H4-VLE Beam Line Instrumentation}

The ProtoDUNE-SP TPC is located in a tertiary extension branch of the $\mathrm{H} 4$ beamline in the CERN North Area. The primary proton beam with a momentum of $400 \mathrm{GeV} / c$ is extracted from the CERN Super Proton Synchrotron (SPS) and is directed towards a beryllium target, producing a 
mixed hadron beam of momentum $80 \mathrm{GeV} / c$. This secondary beam is then transported to impinge on a 'secondary' target, producing a tertiary, very low energy (VLE) beam in the $0.3-7 \mathrm{GeV} / c$ momentum range. The H4-VLE beam line then accepts, momentum-selects and transports these particles to the ProtoDUNE-SP detector. The beam line is equipped with profile monitors, able to provide a measurement of each particle's momentum, trigger counters, able to determine a timeof-flight (TOF) with a total resolution of $\sim 900 \mathrm{ps}$ on a distance of $28.575 \mathrm{~m}$, and two threshold Cherenkov counters, one able to sustain a high-pressure radiator gas (HP), up to 15 bar and a second one able to sustain gases with lower pressures (LP), up to 5 bar [4]. The spectrometer resolution has been extrapolated from MonteCarlo simulation [5], a value of $\Delta p / p \simeq 2.5 \%$ has been determined assuming no material in the beam line for a central momentum of $12 \mathrm{GeV} / c$ and position resolutions of $0.5 \mathrm{~mm}$. When the material along the beam is included, the reconstructed momentum resolution $\Delta p / p$ deteriorates, because of the multiple scattering, with the effect becoming more significant in lower energies $(p<2 \mathrm{GeV} / c)$.

\subsection{Particle Identification}

In a sequence of beam runs, EM showers data from incident electrons of $0.3,0.5,1,2,3,6$ and 7 $\mathrm{GeV}$ energies ( $\pm 5 \%$ typical spread around nominal beam momentum setting) were collected with electron momentum precisely measured on event by event basis by the beam line instrumentation. Information from beam line instrumentation provides particle identification (PID) for the various particle types ( $\mathrm{p}, \mathrm{e}, \pi, \mathrm{k}, \mu$ ) comprising the beam. Depending on the beam momentum (BM) settings, different conditions are applied to the data to select the particle types Table 1.

To solve the remaining ambiguities in the PID, $\pi$ and $\mu$ are distinguished from electrons of 6 and 7 $\mathrm{GeV} / c$, thanks the TPC track reconstruction. Looking at beam events starting shape, the signature given by Pandora, a multi-algorithm approach to automated pattern recognition [6], classifies the events as electrons (showers starting) and $\pi$ and $\mu$ (track starting).

Finally scintillation light spectra analysis allows to recognize $\mu$ from $\pi$, for beam events of 2,3 , 6 , and $7 \mathrm{GeV} / c$. Since muons with momentum $>1.5 \mathrm{GeV} / c$ escape the volume of the TPC, they deposit less energy than pions with a completely different track geometry.

\begin{tabular}{|c|c|c|c|c|c|c|c|}
\hline $\begin{array}{c}6,7 \\
\mathrm{GeV} / c\end{array}$ & $\begin{array}{c}\text { Cherenkov } \\
\text { HP - LP }\end{array}$ & $\begin{array}{c}3 \\
\mathrm{GeV} / \mathrm{c}\end{array}$ & $\begin{array}{c}\text { Cherenkov } \\
\text { HP - LP }\end{array}$ & $\begin{array}{c}2,1,0.5,0.3 \\
\mathrm{GeV} / \mathrm{c}\end{array}$ & $\begin{array}{c}\text { Cherenkov } \\
\text { LP }\end{array}$ & $\begin{array}{c}\text { TOF } \\
2 \mathrm{GeV} / \mathrm{c}\end{array}$ & $\begin{array}{c}\text { TOF } \\
<2 \mathrm{GeV} / c\end{array}$ \\
\hline $\mathrm{e}, \pi, \mu$ & $1-1$ & $\mathrm{e}$ & $1-1$ & $\mathrm{e}$ & 1 & - & - \\
\hline $\mathrm{k}$ & $1-0$ & $\pi, \mu$ & 1 . & $\pi, \mu$ & 0 & $<100 \mathrm{~ns}$ & $<110 \mathrm{~ns}$ \\
\hline $\mathrm{p}$ & $0-0$ & $\mathrm{p}$ & $0-0$ & $\mathrm{p}$ & 0 & $>100 \mathrm{~ns}$ & $>110 \mathrm{~ns}$ \\
\hline
\end{tabular}

Table 1. A summary of the particle identification techniques used in H4-VLE. For higer momentum values $(3,6,7 \mathrm{GeV} / c)$ both threshold Cherenkov counter are active; for lower momentum values $(0.3,0.5,1,2$ $\mathrm{GeV} / c$ ) only the low pressure counter is active. The tag " 1 " means the $\beta$ (velocity) of the particle triggered is higher than the threshold, " 0 " means $\beta$ is lower than threshold. When only one Cherenkov counter is active, the time of flight provides a further PID information. 


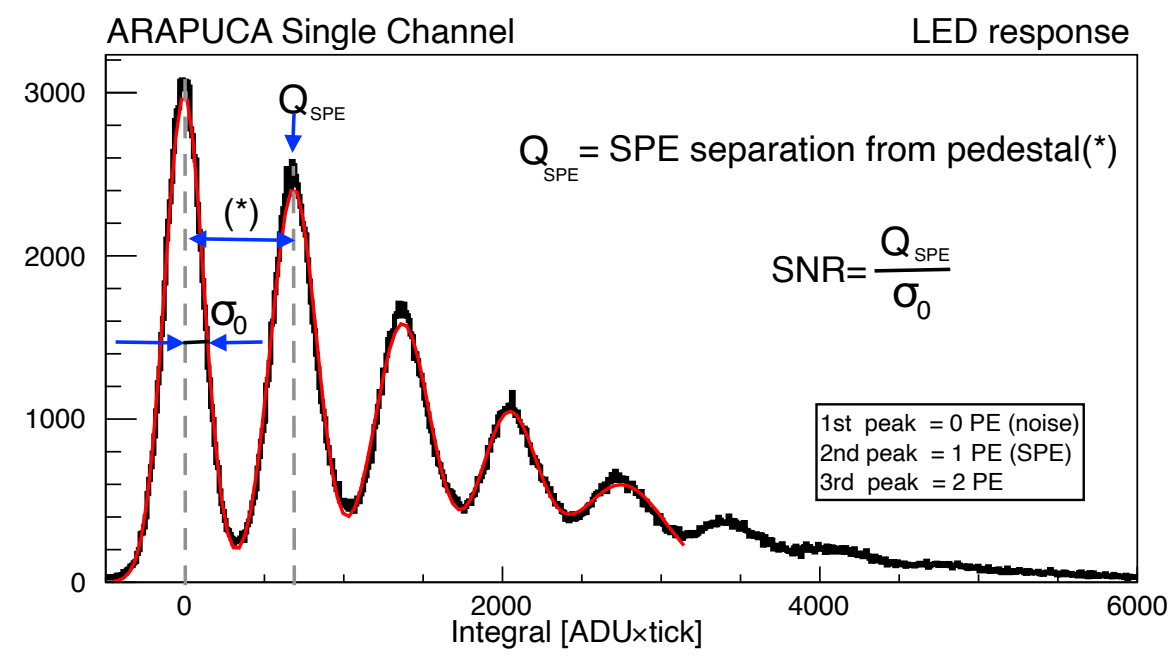

Figure 2. Histogram of the integrated charge (in digital unit) of a single ARAPUCA channel response to LED flashes. The second peak (1-PE) is the single photo-electron response, the first peak (0-PE) width gives the noise level.

\section{Photon Detector Performance}

The ARAPUCA detectors, as well the other PD modules, were calibrated using a pulsed LED, driven inside the TPC trough optical fibers. Each channel has been characterized at various illumination intensity, e.g. figure 2. The single photon response has been quantified as the charge produced per photon detected. Typical gain for the operation bias voltage $(48 \mathrm{~V})$ is $2 \cdot 10^{6}$ electrons per photon detected, measured integrating the recorded signal waveform. Devices have shown a good resolution of the single photon recognition, with a signal-noise ratio $\sim 7$. The signal-to-noise ratio is given by the ratio between the single photo-electron signal (mean value of the second peak fit in figure 2) and the width of the noise (the standard deviation of the first peak fit in figure 2, relative to zero detected photons). Moreover, a study varying the bias voltage was made to characterize all the features of the sensors and to check the stability of the parameters. The 24 channels (12 per ARAPUCA PDM) showed a uniform and stable behavior to all the test performed, none of the parameters were found to be drifting over time for a period of several moths of data taken. Finally, the photo detection efficiency (PDE) has been estimated for the cells of the ARAPUCA module. A set of electrons and muons from beam events with energy 2, 3, 6 and $7 \mathrm{GeV}$ were used to expose and record the response of the detectors to the generated LAr scintillation light. Comparing the number of photons measured and the number of photons estimated to arrive on the detectors by MonteCarlo simulation, a typical value of $(2.05 \pm 0.27) \%$ and $(1.06 \pm 0.10) \%$ of PDE were found (respectively for the ARAPUCA cells whit smaller and larger window surface).

\section{ARAPUCA Photon Detector Response to Beam Electrons}

Operating on the H4-VLE charged particle test beam offered a first ever opportunity to directly probe the calorimetric response with light to EM showers in the sub- to few-GeV/c momentum 

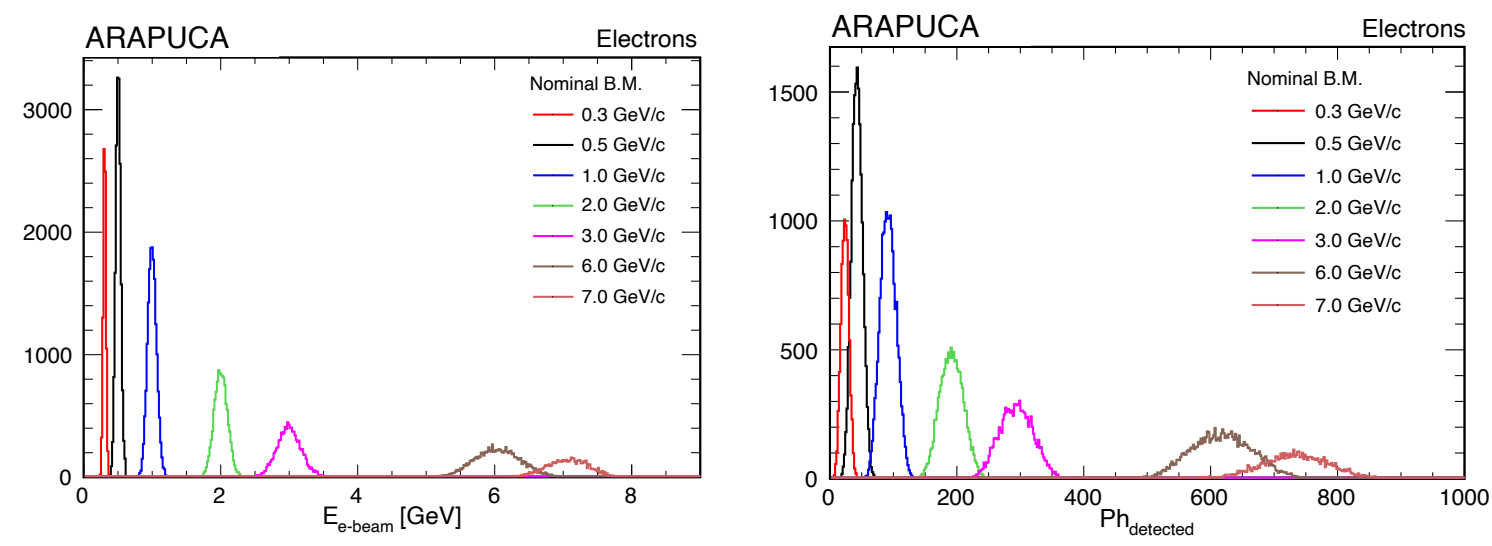

Figure 3. Electron energy distribution (left) and detected photon spectra (right) for the seven nominal beam momentum runs. The detected photon spectra are relative to the photons collected by the whole ARAPUCA PD module.

range. Scintillation photons, collected by the ARAPUCA module in the beam side drift volume, have been analyzed for electrons of $0.3,0.5,1,2,3,6$ and $7 \mathrm{GeV}$. Figure 3 shows the electrons energy distribution (left) and the photons detected spectra (right) for the seven nominal beam momentum values. The observed number of photons have not been corrected for geometry and detection efficiency effects. For each nominal beam momentum data set, a gaussian fit of both distributions gives the average electron energy ${ }^{1}\left\langle E_{e}\right\rangle$ and the corresponding average photon counting $\left\langle N_{p h}\right\rangle$ and their spreads $\left(\sigma_{E}, \sigma_{N}\right)$. Data selection cuts removed events with cosmic ray or spurious beam events pileup.

\subsection{Linearity}

The Gaussian mean value for the photon detected $\left\langle N_{P h}\right\rangle$ vs. the corresponding mean electron energy $\left\langle E_{e}\right\rangle$ is reported in Figure 4 (left). A fit with the function:

$$
N_{P h}=p_{0}+p_{1} \cdot E
$$

clearly shows the linearity of the ARAPUCA's light response to EM energy deposition in LAr over the entire range of energies.

The slope (parameter $p_{1}$ of Eq. (4.1), figure 4 left) gives the light yield: $Y_{\text {light }}=102 \mathrm{Ph} / \mathrm{GeV}$ and a non-zero (negative) y-intercept ( $p_{0}=-8.4 P h$ from the fit) corresponding to an incident energy offset of $-82 \mathrm{MeV}$ compared to nominal for all beam energies. The errors (black vertical bar over point in figure 4 and in the linear fit) are assigned only to the detected photons variable but they take into account both $N_{P h}$ and $E_{e}$ uncertainties. The contributions to the error $\Delta N_{P h}$ come from the error on the mean value given by the fit and the uncertainty with which the number of photons detected and the beam momentum values are measured ${ }^{2}$.

${ }^{1}$ The right quantity to refer to in this study is the electron kinetic energy. At all beam momentum settings electrons are ultra-relativistic and $E_{e} \equiv P_{e} \equiv T_{e}$.

${ }^{2}$ Both the fit mean errors and the uncertainty on the photon detected are negligible; $\Delta N_{P h}$ is mainly coming from the uncertainty on the momentum determination of the beam spectrometer. The data used here are the same in the 

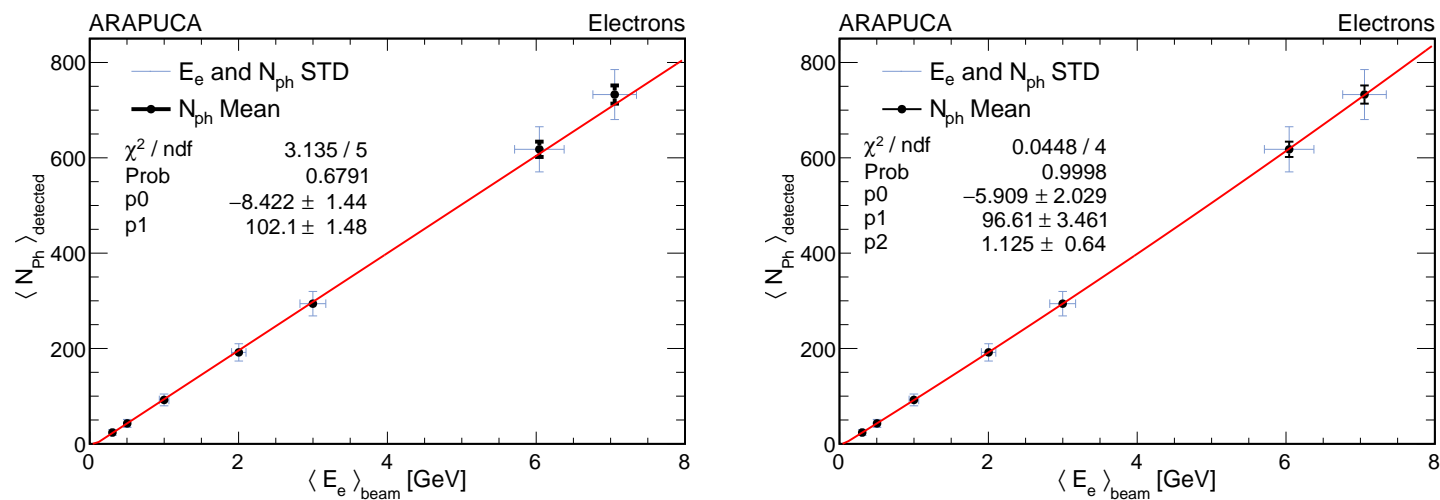

Figure 4. Mean number of detected photons as functions of the mean electron energy [8]. Equation (4.1) is used to fit the black points (left). In light blue are shown the standard deviation values for $N_{p h}$ and $E_{e}$ distributions. Both means and standard deviations come from the Gaussian fit of the distributions in figure 3 . The right plot shows the same data with a fit done adding to Equation (4.1) a term proportional to the square of the energy $\left(p_{2} \cdot E^{2}\right)$.

The light yield value found here is relative to the single ARAPUCA module of the PD system and refers to the specific the beam-to-detector geometry. The total photo-sensitive area of the ARAPUCA module is $\sim 0.05 \%$ of the surface surrounding the LAr volume viewed by the module. The EM shower develops immediately after the beam entry point in the LAr volume at $\sim 3 \mathrm{~m}$ distance in the $\hat{x}$ (drift) direction in front of the ARAPUCA module and nearly parallel to it. The beam in fact is oriented $11.7^{\circ}$ downwards and $10.5^{\circ}$ towards the anode plane where the PD modules are located. Despite the goodness of the linear fit, some deviations from the linearity can be noted, especially at the higher energies. Introducing a term proportional to the square of the energy $\propto E^{2}\left(p_{2} \cdot E^{2}\right)$ in Eq. (4.1), the fit gives a non-negligible value for the second order parameter ( $p_{2}=1.1 \pm 0.6$, figure 4 right). Moreover, from the VLE-H4 beamline studies [4] and MC [5] simulations, beam electrons are expected to loose on average $10-20 \mathrm{MeV}$ in the material in the portion of the beamline downstream the spectrometer and additional $\sim 25-30 \mathrm{MeV}$ while crossing the material inside the cryostat from the end of the beam pipe and the active volume of the TPC (cryostat insulation and membrane, beam tube and a thin LAr layer). The non-zero (negative) y-intercept provides with a direct observation of energy loss in the electron incident beam, whose budget seems overestimated when compared to MC expectation. If instead of the linear fit, a quadratic fit is used ( $E^{2}$ term added), this gives a y-intercept $p_{0}=-5.9$ photons $\rightarrow 61 \mathrm{MeV}$ offset, closer to the MC expected energy loss.

\subsubsection{Geometrical Corrections}

Thanks to the granularity of the ARAPUCA photo-detector module $(\sim 2 \mathrm{~m}$ length along the $\hat{z}$ axis, and segmented in 12 cells-r/o channels), a rather accurate reconstruction of the EM shower spatial/longitudinal development can be made. Looking at the detected "light pattern" by the ARAPUCA cells, i.e. the distribution of the light detected from cell to cell along the module -

ProtoDUNE performance paper [8], the small differences in the $\chi^{2}$ and the linear fit p0 parameter come from adding the uncertainty on the $N_{P h}$ measured. 

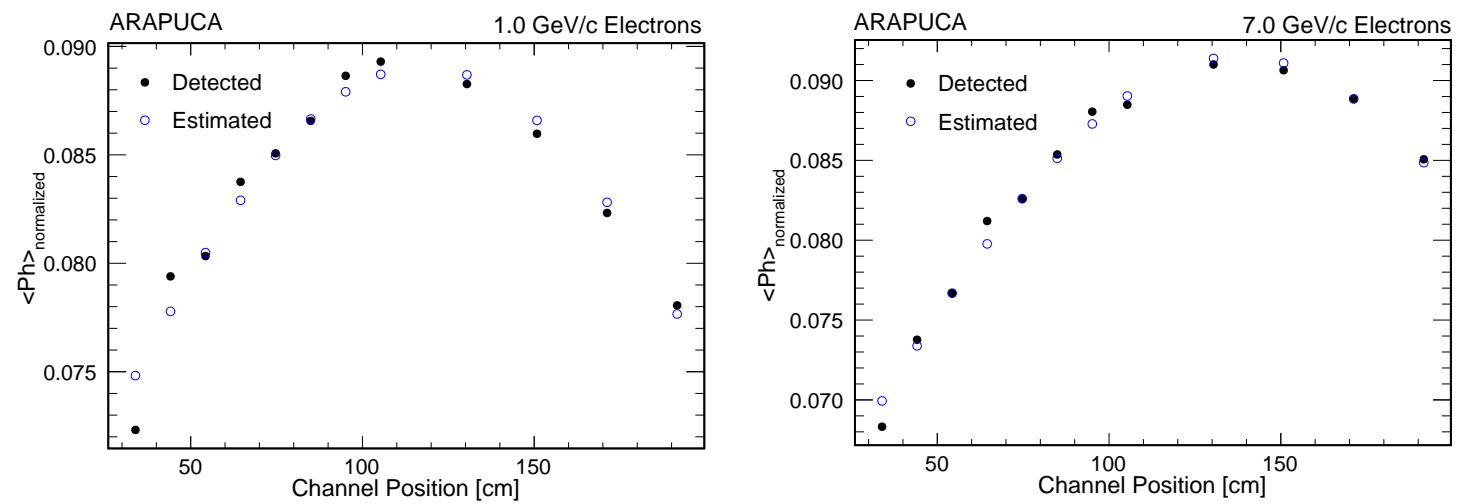

Figure 5. Light pattern on the ARAPUCA PD module, for electromagnetic showers of $1 \mathrm{GeV} / c$ and $7 \mathrm{GeV} / c$ beam momentum. Black points are the average number of photon detected per each cell normalized to their sum. Blue points are the light pattern given by a function chosen through a $\chi^{2}$ minimization approach, having as free parameter a pointlike source moving along the beam direction.
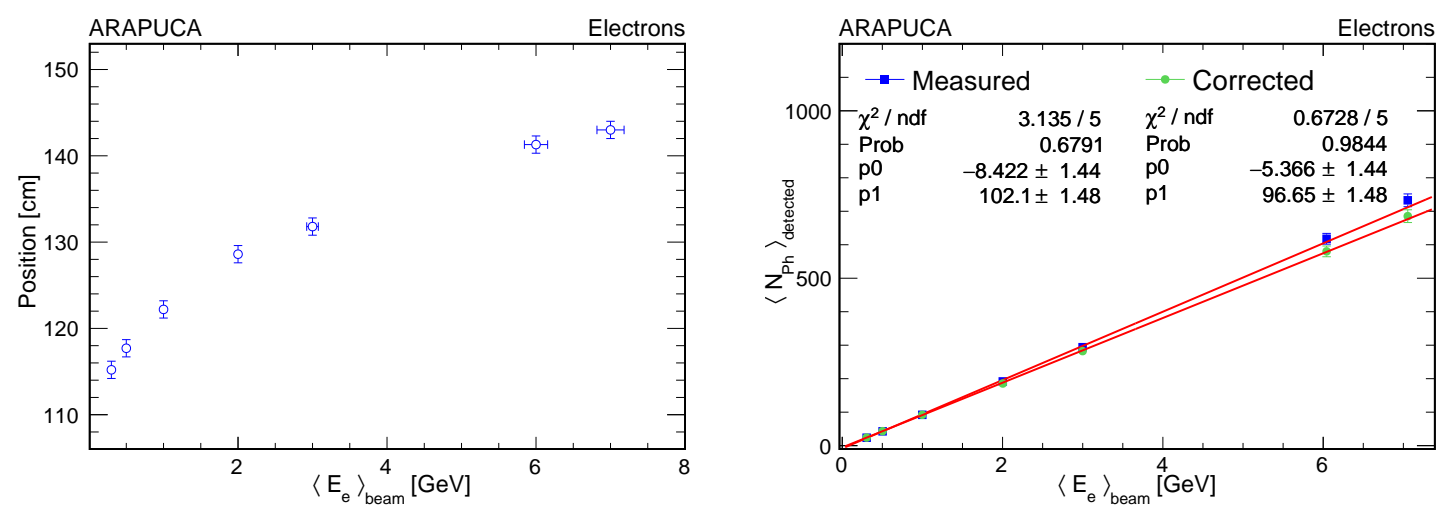

Figure 6. Position of the EM shower light yield maximum along the beam direction (left), as determined from the light patter on the 12 ARAPUCA cells (Figure 5). Linear fit (right) comparing the number of photons detected uncorrected and normalized to the geometry of $1 \mathrm{GeV}$ electrons, given by the position of the maximum reported in the left plot.

figure 5, for $1 \mathrm{GeV}$ EM showers [Left] and for $7 \mathrm{GeV}$ showers [Right], the shower profile and its spacial displacement along $\hat{z}$ at increasing energies are clearly visible (black points).

A simplified model requiring a single parameter is build up to describe the EM shower longitudinal development. It consists of a point-like source moving along the beam direction, (where the distance of the source from the beam entry point is the parameter of the model) and a given function ${ }^{3}$ for the cells geometrical acceptance to reproduce the observed light pattern figure 5 blue points. The choice of the function was driven by obtaining the best similarity to the light pattern given by data, quantified through a $\chi^{2}$ minimization approach. The point-like source

${ }^{3}$ The function found for the model is $f(\vec{r}) \propto \cos \theta_{i} /\left|\vec{r}_{i}\right|^{4}$ (where $\vec{r}_{i}$ is the distance between each cell's center and the point-like source and $\theta_{i}$ is the angle with respect to the perpendicular to the cell's surface). This function is the result of $\chi^{2}$ minimization procedure and it does not come from any physical assumptions. The choice was driven by having a light pattern from a point-like source which best reproduces the data. 
distance from the beam entry point got in this analysis is reported in figure 6 [left]. Based on the light pattern dependence from this parameter a normalization respect one of the given energies can be made.

In figure 6 (right) a comparison between the number of photons detected uncorrected and normalized to the $1 \mathrm{GeV}$ geometry is reported. After these corrections the linear fit slope gives a light yield $Y_{\text {light }}=96.6 \mathrm{Ph} / \mathrm{GeV}$. The $y$-intercept $(p 0=-5.4$ photons from the fit $)$ now corresponds to an incident energy offset of $-56 \pm 14 \mathrm{MeV}$, which is in better agreement with the beam electron energy loss in the materials before entering the TPC from the simulation. Finally the $\chi^{2}$ of the fit has shrunk by a factor of $\sim 5$, indication of a better agreement of the data with a linear behavior. The values found match the constant and linear parameters coming from the second order fit, figure 4 (right). Even if the shower development is clearly not linear whit the energy (figure 6 left), in a first approximation we can remove most of the non-linear effects through this very simple geometric corrections. The new value for the light yield results slightly smaller, but this depends on the choice of the point used for normalization (in this case $1 \mathrm{GeV}$ ).

\subsection{Resolution}
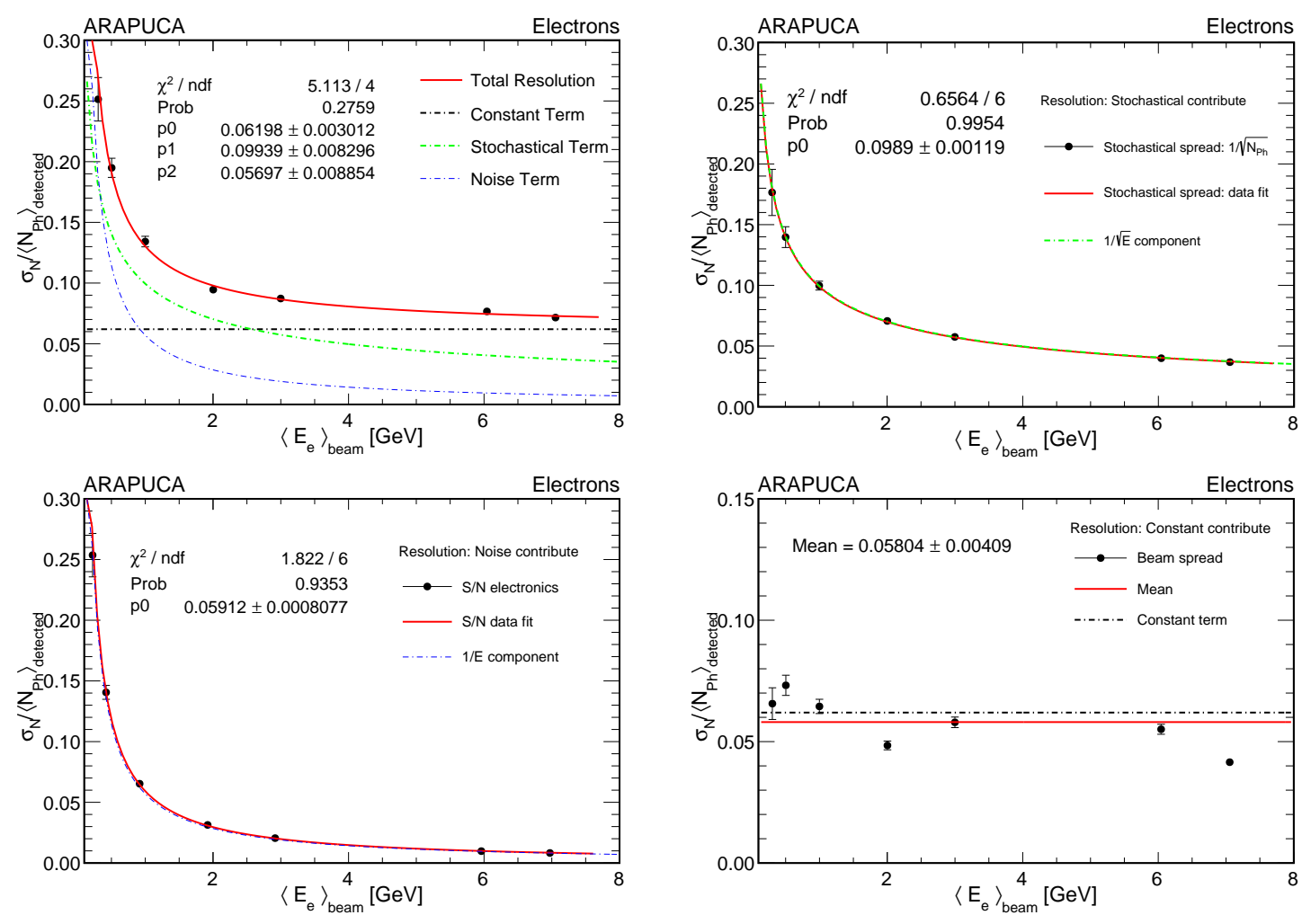

Figure 7. Top left: resolution as a function of $E$ and the three contributions of Eq. (4.2) reported individually: green the stochastic term, blue the noise term and black constant term, as got form the fit. - Top right: $1 / \sqrt{N_{P h}}$ (black point) and their fit (red line) compared with the stochastic term (green line) from the resolution fit. Bottom left: $\sigma_{0} / \mathcal{S}$ (black point) and their fit (red line) compared with the noise term (blue line) from the resolution fit. - Bottom right: $\sigma_{E} / E$ for the beam momentum distribution (black point) and the average value (red line) compared with the constant term (black line) from the resolution fit. 
Based on the linearity of the light response, the relative calorimetric energy resolution $\sigma_{E} / E_{e}$ is obtained from the $\sigma_{N} / N_{p h}$ ratio of the light response. The energy resolution, as for a homogeneous calorimeter, can be expressed in a general form [7] depending on three different contributions:

$$
\frac{\sigma_{E}}{E}=\sqrt{p_{0}^{2}+\left(\frac{p_{1}}{\sqrt{E}}\right)^{2}+\left(\frac{p_{2}}{E}\right)^{2}}
$$

The terms on the right-hand side of Eq. (4.2) are referred to below as the "constant term", the "stochastic term" and the "noise term", figure 7 top left. The contributions, Eq. (4.2), to the resolution can be split (figure 7), analyzed individually and compared with the supposed relative source. Values after the geometric corrections are not reported since the resolution resulted to be not sensitive to such small corrections. In the other hand, the amount of energy loss before entering the TPC, given by the $y$-intercept of the linear fit, was not negligible. To take into account that, correction for -5.4 photons has been made ( $p_{0}$ form the corrected values fit, figure 6 right).

- The stochastic contribution comes from the intrinsic spread in the distribution of the number of photons detected given by the Poisson statistics, $\sigma_{P h}=\sqrt{N_{P h}}$ :

$$
\left(\frac{\sigma_{P h}}{N_{P h}}\right)_{\text {Stochastic }}=\frac{1}{\sqrt{N_{P h}}} \propto \frac{1}{\sqrt{E}}
$$

Figure 7 (top right) shows $1 / \sqrt{N_{P h}}$ as a function of the beam energy compared with the stochastic contribution in the energy resolution. The fit of this quantity, using the function $f(x)=p 0 / \sqrt{x}$, gives for the parameter value $(9.89 \pm 0.12) \%$, which is in good agreement with the value from the energy resolution fit for the stochastic term $p_{1}=(9.94 \pm 0.83) \%$, (parameter $p_{1}$ in Eq. (4.2) and figure 7 top left).

- The noise contribution comes from the electronic noise of the readout chain. For each energy, the noise contribution is obtained from the quadratic sum of the ratio between the noise and the average signal of each channel:

$$
\left(\frac{\sigma_{P h}}{N_{P h}}\right)_{\text {Noise }}=\sqrt{\sum_{i}^{12}\left(\frac{\sigma_{0 i}}{\mathcal{S}_{i}}\right)^{2}}=\sqrt{\sum_{i}^{12}\left(\frac{\sigma_{0 i}}{Q_{S P E_{i}}} \frac{1}{\left\langle N_{P h}\right\rangle_{i}}\right)^{2}}
$$

where for each i-th channel: $\sigma_{0 i}$ is the standard deviation of the noise (first peak in figure 2), $Q_{S P E_{i}} / \sigma_{0 i}$ is the signal-to-noise ratio (where $Q_{S P E_{i}}$ is the charge produced by a single photo-electron, given by the mean of the second peak in figure 2) and $\mathcal{S}_{i}=\left\langle N_{P h}\right\rangle_{i} \cdot Q_{S P E_{i}}$ is the amplitude of the signal (in terms of charge) relative to the average number of photon detected. A fit of the noise using the function $f(x)=p 0 / x$, gives a parameter value of $(59 \pm 1) \mathrm{MeV}$ (figure 7 bottom left), compatible with the the noise term from the energy resolution fit $p_{2}=(57 \pm 9) \mathrm{MeV}$, (parameter $p_{2}$ in Eq. (4.2), figure 7 top left).

- The constant term may find contributions from different sources. The main one is identified in the beam electron energy spread $\sigma_{e} / E$ (distributions shown in figure 3 left). Values are reported in figure 7 bottom right, (black points). The mean value $(5.8 \pm 0.4) \%$ is shown with red line. The beam spread is a significant contribution to the constant term $p_{0}=(6.2 \pm 0.3) \%$ 
from the resolution fit (parameter $p_{0}$ in Eq. (4.2), figure 7 top left). An additional contribution to the constant term comes from fluctuations in the energy loss in the materials before electrons enter the TPC. Since it happens downstream the last momentum spectrometer, this energy degradation and its fluctuation do not appear in the energy spectra (figure 3 left) and it is evaluated by simulation. For $1 \mathrm{GeV}$ electrons the uncertainty on the energy loss is $\sim 2 \%$, increasing at lower energies [5]. The quadratic sum of the measured spread in the beam energy (5.8\% figure 7 bottom right) and the simulated additional fluctuations in the energy loss downstream the spectrometer (2\%), give a value of $6.1 \%$, compatible with the constant term of the resolution fit $p_{0}=(6.2 \pm 0.3) \%$.

Other contributions to the resolution, such as non-uniformity on the detector illumination, channel to channel response variation and possible shower leakage across the cathode, have been investigated, resulting negligible.

\section{Conclusion}

The ARAPUCA PD module installed in ProtoDUNE has shown excellent calorimetric response to beam electrons light signals. Linearity over the entire range of energies analyzed is observed. Despite its relative small photo-sensitive area and the large distance from the light source, a good resolution has been observed, and explained in terms of the components which contribute to the spread in the detected photon response .

\section{References}

[1] The Single-Phase ProtoDUNE Technical Design Report,CERN-SPSC-2017-028 / SPSC-TDR-005 (June 19, 2017).

[2] A.A. Machado and E. Segreto, ARAPUCA a new device for liquid argon scintillation light detection,Instrum. 11 (2016) C02004.

[3] T. Anderson, P. De Lurgio, Z. Djurcic, G. Drake, A. Kreps, M. Oberling, LBNE SiPM Signal Processor User Manual, Argonne National Laboratory, October 27, 2015.

[4] A.C. Booth, N. Charitonidis, P. Chatzidaki, Y. Karyotakis, Nowak, I. Ortega-Ruiz, M. Rosenthal and P. Sala, Particle production, transport, and identification in the regime of $1-7 \mathrm{GeV} / \mathrm{c}$, PHYSICAL REVIEW ACCELERATORS AND BEAMS 22, 061003 (2019).

[5] N. Charitonidis, Y.Karyotakis, and I. Efthymiopoulos, Beam performance and instrumentation studies for the ProtoDUNE-DP experiment of CENF, CERN-ACC-NOTE-2016-0052.

[6] J. S. Marshall, M. A. Thomson, The Pandora Software Development Kit for Pattern Recognition,Eur. Phys. J. C75 (2015) 439, [1506.05348].

[7] W. Fabjan and F. Gianotti Calorimetry for Particle Physics, CERN-EP/2003-075 (CERN, 1211 Geneva 23, Switzerland).

[8] DUNE collaboration, First results on ProtoDUNE-SP LArTPC performance from a test beam run at the CERN Neutrino Platform, to be submitted to JINST 